\title{
Computation of Nonlinear Functionals in Particle Methods
}

\author{
Axel Klar
}

Fachbereich Mathematik

Universität Kaiserslautern

Postfach 3049

67663 Kaiserslautern, FRG

E-Mail: klar@mathematik.uni-kl.de

AMS Subject Classification: 65C05, 65D15

March 1995 


\title{
Computation of Nonlinear Functionals in Particle Methods
}

\author{
by AXEL KLAR
}

\begin{abstract}
Zusammenfassung
Computation of Nonlinear Functionals in Particle Methods. We consider the numerical computation of nonlinear functionals of distribution functions approximated by point measures. Two methods are described and estimates for the speed of convergence as the number of points tends to infinity are given. Moreover numerical results for the entropy functional are presented.

Berechnung von nichtlinearen Funktionalen bei Partikelmethoden. Wir betrachten die numerische Berechnung nichtlinearer Funktionale von Verteilungsfunktionen, die durch Punktmaße angenähert werden. Zwei Methoden werden beschrieben und Abschätzungen für die Konvergenzgeschwindigkeit werden angegeben. Außerdem werden numerische Resultate für das Entropiefunktional vorgestellt.
\end{abstract}

\section{Introduction}

Particle methods are widely used for the numerical solution of high dimensional evolution equations, e.g. the Boltzmann equation in the kinetic theory of gases, see e.g. [?, ?, ?, ?, ?] for convergence proofs, applications, reviews and further references. The solutions of these equations are usually density functions which can be approximated by particle ensembles. It is well known that linear functionals of these density functions, e.g. moments, are computed to a good accuracy with a reasonable number of particles. One has simply to compute the empirical averages. For estimates see e.g. Kuipers and Niederreiter [?]. The situation is different if one wants to get a good approximation of the distribution function itself or of nonlinear functionals. This is to a sufficient degree of accuracy only possible with a very large number of particles.

Usually a coarse graining of the particle space is employed to approximate the density function such that one is able to compute the required integrals. We give in Section 2 an error estimate for the approximation of nonlinear functionals by this method.

In Section 3 we try to avoid the drawbacks of the coarse-graining method - high particles numbers, arbitrariness in choosing the coarse graining - by assuming that the density 
function is out of a certain class of functions. This class is prescribed by the physical situation under consideration. Estimates that show the improvement in speed of convergence compared to the coarse graining method can then be given.

In Section 4 numerical examples are given. We compute the entropy for different types of distribution functions occuring in kinetic theory, Maxwell, Navier Stokes, and Mott Smith distributions. Moreover the time development of the entropy for the BKW solution of the homogeneous Boltzmann equation is shown.

\section{Coarse Graining}

Let $f: \mathbb{R} \rightarrow \mathbb{R}^{+}$be a density function, i.e. $f$ is positive and $\mathcal{L}^{1}(\mathbb{R})$-integrable with $\int_{\mathbb{R}} f(v) d v=1$. We write $f \in \mathcal{L}_{+}^{1,1}(\mathbb{R})$. The particle ensembles $\left\{\left(v_{1}, \ldots, v_{N}\right)\right\}_{N \in \mathbb{N}}$ with $v_{j} \in \mathbb{R}, j=1 \ldots N$, approximate $f$, if the measure $\omega_{N}:=\frac{1}{N} \sum_{i=1}^{N} \delta\left(v_{i}-v\right), N \in \mathbb{N}$ generated by the above pointsets converge weakly in the measure theoretic sense to $f(v) d v$. Here $\delta\left(v_{i}-v\right)$ denotes the Dirac measure at the point $v_{i}$. We write $\omega_{N} \stackrel{w}{\rightarrow} f$ as $N \rightarrow \infty$. It is equivalent to requiring that the discrepancy $D\left(\omega_{N}, f\right)$ tends to 0 as $N$ tends to infinity with

$$
D\left(\omega_{N}, f\right):=\sup _{a<b}\left|\frac{1}{N} \sum_{j=1}^{N} \chi_{[a, b)}\left(v_{j}\right)-\int_{[a, b)} f(v) d v\right| .
$$

Here we denoted by $\chi_{[a, b)}$ the characteristic function of the interval $[a, b) \subset \mathbb{R}$. The equivalence was shown already by Weyl [11], see Neunzert/Wick [9] for the multidimensional case.

As a first step we approximate the density function $f$ in a strong sense. Let $\left\{\Delta_{i}^{(n)}\right\}_{i \in \mathbb{Z}}$ be an equidistant partition of $\mathbb{R}$ into intervals $\Delta_{i}^{(n)}=\left[a_{i}^{(n)}, b_{i}^{(n)}\right)$ of size $\left|\Delta_{i}^{(n)}\right|=\frac{1}{n}$, $\Delta_{i}^{(n)} \cap \Delta_{j}^{(n)}=\emptyset, i \neq j, \bigcup_{i \in \mathbb{Z}} \Delta_{i}^{(n)}=\mathbb{R}$. Then we define for $v \in \mathbb{R}$ and $\Delta_{i}^{(n)}(v)$ the interval of the above partition containing $v$

$$
\begin{aligned}
P_{n} \omega_{N}(v) & :=\frac{1}{\left|\Delta_{i}^{(n)}(v)\right|} \omega_{N}\left(\Delta_{i}^{(n)}(v)\right) \\
& =\frac{n}{N} \sum_{j=1}^{N} \chi_{\Delta_{i}^{(n)}(v)}\left(v_{j}\right) .
\end{aligned}
$$

Analogously

$$
P_{n} f(v):=n \int_{\Delta_{i}^{(n)}(v)} f\left(v^{\prime}\right) d v^{\prime}
$$

In the next lemma we give an error estimate for the approximation of $f$ by $P_{n} \omega_{N}$ as $n$ and $N$ tend to infinity. 
Lemma 2.1 Let $f \in \mathcal{L}_{+}^{1,1}(\mathbb{R})$ be Lipschitz continuous with Lipschitz constant $L$ and $\omega_{N}$ point measures approximating $f$, i.e. $\omega_{N} \stackrel{w}{\rightarrow} f$, then we have $\forall v \in \mathbb{R}$ :

$$
\left|P_{n} \omega_{N}(v)-f(v)\right| \leq \frac{L}{n}+n D\left(\omega_{N}, f\right)
$$

In particular $P_{n} \omega_{N} \rightarrow f$ pointwise in $\mathbb{R}$, if $n, N \rightarrow \infty$ and $D\left(\omega_{N}, f\right)$ tends faster to 0 than $\frac{1}{n}$.

Remark: Pointsets $\left\{\left(v_{1}, \ldots, v_{N}\right)\right\}_{N \in \mathbb{N}}$ can be easily constructed for any $f \in \mathcal{L}_{+}^{1,1}(\mathbb{R})$ such that $D\left(\omega_{N}, f\right) \sim \frac{1}{N}$. This rate is optimal. If $\left\{\left(v_{1}, \ldots, v_{N}\right)\right\}_{N \in \mathbb{N}}$ is generated by a sequence of points $v_{1}, \ldots, v_{N}, \ldots$, then the optimal convergence rate ist $D\left(\omega_{N}, f\right) \sim \frac{\ln N}{N}$, see Kuipers et al. [6].

This means that for $n$ tending to infinity like $N^{k}, 0<k<1$ we get $P_{n} \omega_{N} \rightarrow f$. In contrast if $n \sim N^{k}, k \geq 1$ it is easy to construct a counterexample even for points with an optimal convergence rate.

\section{Proof:}

$$
\begin{aligned}
& \left|P_{n} \omega_{N}(v)-f(v)\right| \leq\left|P_{n} \omega_{N}(v)-P_{n} f(v)\right|+\left|P_{n} f(v)-f(v)\right| \\
& \leq \sum_{i \in \mathbb{Z}} \chi_{\Delta_{i}^{(n)}}(v)\left[\left|\frac{n}{N} \sum_{j=1}^{N} \chi_{\Delta_{i}^{(n)}}\left(v_{j}\right)-n \int_{\Delta_{i}^{(n)}} f\left(v^{\prime}\right) d v^{\prime}\right|+\left|n \int_{\Delta_{i}^{(n)}} f\left(v^{\prime}\right) d v^{\prime}-f(v)\right|\right] \\
& \leq \sum_{i \in \mathbb{Z}} \chi_{\Delta_{i}^{(n)}}(v)\left[n D\left(\omega_{N}, f\right)+\left|n \int_{\Delta_{i}^{(n)}} f\left(v^{\prime}\right) d v^{\prime}-n \int_{\Delta_{i}^{(n)}} f(v) d v^{\prime}\right|\right] \\
& \leq \sum_{i \in \mathbb{Z}} \chi_{\Delta_{i}^{(n)}}(v)\left[n D\left(\omega_{N}, f\right)+n \int_{\Delta_{i}^{(n)}}\left|f\left(v^{\prime}\right)-f(v)\right| d v^{\prime}\right] \\
& \leq n D\left(\omega_{N}, f\right)+L \cdot \frac{1}{n}
\end{aligned}
$$

We use this approximation to compute nonlinear functionals of $f$. Let $f$ be Lipschitz continuous and in $\mathcal{L}_{+}^{1,1}(\mathbb{R})$ as before. Moreover we assume $f$ to be 0 outside of $B_{R}:=$ $\{v \in \mathbb{R}|| v \mid \leq R\}, R>0$ and the approximating points $\left\{\left(v_{1}, \ldots, v_{N}\right)\right\}_{N \in \mathbb{N}}, \omega_{N} \stackrel{w}{\rightarrow} f$ to be in $B_{R}$. According to the above lemma we assume also that $n D\left(\omega_{N}, f\right)$ tends to 0 . The assumptions on $f$ are not really necessary: we could have also assumed $f$ to decay fast enough as $v$ tends to infinity.

We are interested in functionals of the form

$$
\int \phi(f(v)) d v
$$


where $\phi: \mathbb{R}^{+} \rightarrow \mathbb{R}$ is continuous and $\phi(0)=0$. Define for $h>0$ and $C>0$ the modulus of continuity

$$
M_{\phi}^{C}(h):=\sup \{|\phi(x)-\phi(y)||x, y \in[0, C],| x-y \mid \leq h\} .
$$

\section{Proposition 2.2}

$$
\begin{aligned}
& \left|\int \phi(f(v)) d v-\sum_{i \in \mathbb{Z}} \frac{1}{n} \phi\left(\frac{n}{N} \sum_{j=1}^{N} \chi_{\Delta_{i}^{(n)}}\left(v_{j}\right)\right)\right| \\
& \leq C_{f} M_{\phi}^{C_{f}}\left(\frac{L}{n}+n D\left(\omega_{N}, f\right)\right)
\end{aligned}
$$

where $C_{f}$ is a constant depending on $f$.

\section{Proof:}

$$
\begin{aligned}
& \left|\int \phi(f(v)) d v-\sum_{i \in \mathbb{Z}} \frac{1}{n} \phi\left(\frac{n}{N} \sum_{j=1}^{N} \chi_{\Delta_{i}^{(n)}}\left(v_{j}\right)\right)\right| \\
& =\left|\int \phi(f(v)) d v-\int \phi\left(P_{n} \omega_{N}(v)\right) d v\right| \\
& \leq \int\left|\phi(f(v)) d v-\phi\left(P_{n} \omega_{N}(v)\right)\right| d v \\
& =\int P_{B_{\tilde{R}}}\left|\phi(f(v))-\phi\left(P_{n} \omega_{N}(v)\right)\right| d v \\
& \leq \sum_{i \in I_{n}^{\tilde{R}} \Delta_{i}^{(n)}} \int_{n}\left|\phi(f(v))-\phi\left(P_{n} \omega_{N}(v)\right)\right| d v
\end{aligned}
$$

if $\tilde{R}$ is chosen large enough and $I_{n}^{\tilde{R}}:=\left\{i \in \mathbb{Z} \mid \Delta_{i}^{(n)} \cap B_{\tilde{R}} \neq \emptyset\right\}$.

Using the Lipschitz property of $f$, Lemma 2.1, the definition of $M_{\phi}^{C}$ and the fact that $n D\left(\omega_{N}, f\right)$ is bounded by a constant depending only on $f$, one obtains that this is smaller than

$$
\sum_{i \in I_{n}^{\tilde{R}}} \frac{1}{n} M_{\phi}^{C_{f}}\left(\frac{L}{n}+n D\left(\omega_{N}, f\right)\right),
$$

$C_{f}$ a constant depending on $f$. Finally the last term is estimated from above by

$$
C_{f} M_{\phi}^{C_{f}}\left(\frac{L}{n}+n D\left(\omega_{N}, f\right)\right)
$$

$C_{f}$ some constant depending on $f$.

Remark: We remark that the conditions on $\phi$ are fulfilled for example if $\phi(x)=x^{p}, p \in \mathbb{N}$ or for the entropy functional, i.e. $\phi(x)=-x \ln x$, if $x>0$ and $\phi(x)=0$, if $x=0$. Moreover we have for these choices of $\phi$ by an easy computation

$$
M_{\phi}^{C}(h) \leq A h
$$


and

$$
M_{\phi}^{C}(h) \leq A h|\ln h|
$$

respectively, for $h$ small and $A$ a constant depending on $C$.

This means that $M_{\phi}^{C}$ tends to 0 for $h$ tending to 0 with a certain rate. Choosing e.g. an optimal sequence of points $v_{1}, \ldots, v_{N}, \ldots$ s.t. $D\left(\omega_{N}, f\right) \sim \frac{\ln N}{N}$ and $n=\left(\frac{N}{\ln N}\right)^{\frac{1}{2}}$, we get for the entropy functional convergence rates for the approximation in Proposition 2.2 proportional to $\frac{(\ln N)^{3 / 2}}{N^{1 / 2}}$.

\section{Hermite Expansions}

Having a certain preknowledge of the density function $f$, for example that $f$ is out of a special class of functions, one can use more efficient methods to compute nonlinear functionals. E.g. in kinetic theory one is often particularly interested in situations close to thermal equilibrium. This means that the density function can be expanded around a local Maxwellian distribution. Grad [4] uses such a higher order expansion in Hermite functions to describe the flow properties. A discussion of this approach can be found in Cercignani [3].

We proceed here in the same way. Let $H_{k}(v), k \in \mathbb{N}^{0}$ be defined by $H_{k}(v)=M^{\frac{1}{2}}(v) \frac{1}{\sqrt{k !}} \tilde{H}_{k}(v)$, where $M(v):=\frac{1}{\sqrt{2 \pi}} e^{-\frac{v^{2}}{2}}$ and $\tilde{H}_{k}(v)=(-1)^{k} e^{\frac{v^{2}}{2}}\left(\frac{\partial}{\partial v}\right)^{k} e^{-\frac{v^{2}}{2}}$. Any $f$ in $\mathcal{L}^{2}(\mathbb{R})$ can be expanded as $f=\sum_{k=0}^{\infty}<f, H_{k}>H_{k}$ with $<,>$ the usual scalarproduct in $\mathcal{L}^{2}(\mathbb{R})$. We assume that the density function $f \in \mathcal{L}^{2}(\mathbb{R})$ can be represented by a finite series of Hermite functions

$$
f=\sum_{k=0}^{K}<f, H_{k}>H_{k}, K \in \mathbb{N}^{0} .
$$

Let $\left\{\left(v_{1}, \ldots, v_{N}\right)\right\}_{N \in \mathbb{N}}$ denote again pointsets approximating $f, \omega_{N} \stackrel{w}{\rightarrow} f . a_{k}\left(\omega_{N}\right)$ is defined as $a_{k}\left(\omega_{N}\right):=\frac{1}{N} \sum_{j=1}^{N} H_{k}\left(v_{j}\right)$. Then we have

\section{Lemma 3.1}

$$
\left|f(v)-\sum_{k=0}^{K} a_{k}\left(\omega_{N}\right) H_{k}(v)\right| \leq M_{K} D\left(\omega_{N}, f\right)
$$

$\forall v \in \mathbb{R}$, where $M_{K}$ is a constant depending on $K$.

\section{Proof:}

$$
\left|f(v)-\sum_{k=0}^{K} a_{k}\left(\omega_{N}\right) H_{k}(v)\right|
$$




$$
\begin{aligned}
& \leq\left|\sum_{k=0}^{K}\left(<f, H_{k}>-a_{k}\left(\omega_{N}\right)\right) H_{k}(v)\right| \\
& \leq \sup _{k \in(0 \ldots K)}\left(\left|<H_{k}, f>-a_{k}\left(\omega_{N}\right)\right|\right) \cdot \sup _{v \in \mathbb{R}} \sum_{k=0}^{K}\left|H_{k}(v)\right|
\end{aligned}
$$

Using the Koksma-Hlavka inequality, see Kuipers et al. [6], we get

$$
\left|<H_{k}, f>-a_{k}\left(\omega_{N}\right)\right| \leq V\left(H_{k}\right) D\left(\omega_{N}, f\right)
$$

where $V\left(H_{k}\right)$ is the variation of $H_{k}$ with

$$
V\left(H_{k}\right)=\int\left|H_{k}^{\prime}(v)\right| d v
$$

This yields

$$
\begin{aligned}
& \left|f(v)-\sum_{k=0}^{K} a_{k}\left(\omega_{N}\right) H_{k}(v)\right| \\
& \leq K \cdot \sup _{k \in(0, \ldots, K)}\left(\int\left|H_{k}^{\prime}(v)\right| d v\right) \cdot \sup _{k \in(0, \ldots, K)}\left(\sup _{v \in \mathbb{R}}\left(\left|H_{k}(v)\right|\right)\right) \cdot D\left(\omega_{N}, f\right) \\
& =M_{K} D\left(\omega_{N}, f\right)
\end{aligned}
$$

We turn to the computation of the functionals $\int \phi(f(v)) d v$ with $\phi: \mathbb{R}^{+} \rightarrow \mathbb{R}$ continuous and $\phi(0)=0$. Consider at the beginning the special case $\phi(x)=x^{2}, x \geq 0$. With a density function $f \in \mathcal{L}^{2}(\mathbb{R}), f=\sum_{k=0}^{K}<H_{k}, f>H_{k}, \forall v \in \mathbb{R}$, we get, using the $\mathcal{L}^{2}(\mathbb{R})$ orthogonality of $H_{k}, k \in \mathbb{N}^{0}$ :

\section{Proposition 3.2}

$$
\left|\int f^{2}(v) d v-\sum_{k=0}^{K} a_{k}^{2}\left(\omega_{N}\right)\right| \leq C_{f} D\left(\omega_{N}, f\right)
$$

where $C_{f}$ is a constant depending on $f$.

\section{Proof:}

$$
\begin{aligned}
& \left|\int f^{2}(v) d v-\sum_{k=0}^{K} a_{k}^{2}\left(\omega_{N}\right)\right| \\
& =\left|\int f^{2}(v) d v-\int\left(\sum_{k=0}^{K} a_{k}\left(\omega_{N}\right) H_{k}(v)\right)\left(\sum_{k=0}^{K} a_{k}\left(\omega_{N}\right) H_{k}(v)\right) d v\right|
\end{aligned}
$$




$$
\begin{aligned}
& \leq \int\left|f^{2}(v)-\left(\sum_{k=0}^{K} a_{k}\left(\omega_{N}\right) H_{k}(v)\right)^{2}\right| d v \\
& =\int\left|f(v)+\sum_{k=0}^{K} a_{k}\left(\omega_{N}\right) H_{k}(v)\right| \cdot\left|f(v)-\sum_{k=0}^{K} a_{k}\left(\omega_{N}\right) H_{k}(v)\right| d v \\
& \leq M_{K} D\left(\omega_{N}, f\right) \int\left|f(v)+\sum_{k=0}^{K} a_{k}\left(\omega_{N}\right) H_{k}(v)\right| d v
\end{aligned}
$$

where we used Lemma 3.1 This is smaller than

$$
\begin{aligned}
& M_{K} D\left(\omega_{N}, f\right)\left(\int|f(v)| d v+\sum_{k=0}^{K}\left|a_{k}\left(\omega_{N}\right)\right| \int\left|H_{k}(v)\right| d v\right) \\
& \leq C_{f} D\left(\omega_{N}, f\right),
\end{aligned}
$$

$C_{f}$ a constant depending on $f$

We consider now the computation of more general functionals with $\phi$ as assumed above. For simplicity we restrict the class of density functions $f \in \mathcal{L}_{+}^{1,1}(\mathbb{R})$ to the following: $f$ is

0 outside some ball $B_{R}=\{v \in \mathbb{R}|| v \mid \leq R\}, R>0$ and $f(v)=\sum_{k=0}^{K}<H_{k}, f>H_{k}(v), \forall v \in$ $B_{R}$. Moreover the approximating points $\left\{\left(v_{1}, \ldots, v_{N}\right)\right\}_{N \in \mathbb{N}}, \omega_{N} \stackrel{w}{\rightarrow} f$ are assumed to be in $B_{R}$ as before.

Let $\left\{\Delta_{i}^{h}\right\}_{i \in \mathbb{Z}}, h>0$ be a fixed partition of $\mathbb{R}$ into intervals $\Delta_{i}^{h}$ with $\left|\Delta_{i}^{h}\right|=h$ so that $\Delta_{i}^{h} \cap \Delta_{j}^{h}=\emptyset$ and $\bigcup_{i \in I_{h}^{R}} \Delta_{i}^{h}=B_{R}, I_{h}^{R} \subset \mathbb{Z}$.

\section{Proposition 3.3}

$$
\begin{aligned}
& \left|\int \phi(f(v)) d v-\sum_{i \in I_{h}^{R}} h \phi\left(\sum_{k=0}^{K} a_{k}\left(\omega_{N}\right) H_{k}\left(\xi_{i}\right)\right)\right| \\
& \leq C_{f} M_{\phi}^{C_{f}}\left(L h+M_{K} D\left(\omega_{N}, f\right)\right)
\end{aligned}
$$

where $C_{f}$ is a constant depending on $f, M_{K}$ depends on $K$ and $L$ is the Lipschitz constant of $f$ on $B_{R}$. $\xi_{i}$ is a fixed value, $\xi_{i} \in \Delta_{i}^{h}, i \in I_{h}^{R} \subset \mathbb{Z}$.

Remark: Comparing this estimate to the one in Proposition 2.2 we see that the number of particles and the size $h \sim \frac{1}{n}$ of the partitions is decoupled.

Moreover the values $H_{k}\left(\xi_{i}\right)$ can be computed numerically in advance such that only the computation of the moments is left.

\section{Proof:}

$$
\left|\int \phi(f(v)) d v-\sum_{i \in I_{h}^{R}} h \phi\left(\sum_{k=0}^{K} a_{k}\left(\omega_{N}\right) H_{k}\left(\xi_{i}\right)\right)\right|
$$




$$
\begin{aligned}
& =\left|\sum_{i \in I_{h}^{R}} \int_{\Delta_{i}^{h}}\left[\phi(f(v))-\phi\left(\sum_{k=0}^{K} a_{k}\left(\omega_{N}\right) H_{k}\left(\xi_{i}\right)\right)\right] d v\right| \\
& \leq \sum_{i \in I_{h}^{R}} \int_{\Delta_{i}^{h}}\left|\phi(f(v))-\phi\left(\sum_{k=0}^{K} a_{k}\left(\omega_{N}\right) H_{k}\left(\xi_{i}\right)\right)\right| d v \\
& \leq \sum_{i \in I_{h}^{R}}\left[\int_{\Delta_{i}^{h}}\left|\phi(f(v))-\phi\left(f\left(\xi_{i}\right)\right)\right| d v+\int_{\Delta_{i}^{h}}\left|\phi\left(f\left(\xi_{i}\right)\right)-\phi\left(\sum_{k=0}^{K} a_{k}\left(\omega_{N}\right) H_{k}\left(\xi_{i}\right)\right)\right| d v\right]
\end{aligned}
$$

Using the special form of $f$ we see that it is Lipschitz on $B_{R}$ and we get for all $v \in \Delta_{i}^{h}$ and $L$ the Lipschitz constant of $f$ on $B_{R}$

$$
\left|f(v)-f\left(\xi_{i}\right)\right| \leq L h .
$$

This means that the above is smaller than

$$
\sum_{i \in I_{h}^{R}}\left[h M_{\phi}^{C_{f}}(L h)+h M_{\phi}^{C_{f}}\left(M_{K} D\left(\omega_{N}, f\right)\right)\right]
$$

where we used Lemma 3.1. $C_{f}$ is a constant depending on $f$. The last term is estimated by

$$
C_{f} M_{\phi}^{C_{f}}(L h)+C_{f} M_{\phi}^{C_{f}}\left(M_{K} D\left(\omega_{N}, f\right)\right)
$$

with $C_{f}$ sufficiently large.

Remark: By the estimates on $M_{\phi}^{C}$ in the last section we get for the entropy functional, using an optimal point sequence $v_{1}, \ldots, v_{N}, \ldots$ with $D\left(\omega_{N}, f\right) \sim \frac{\ln N}{N}$ and $h=\frac{\ln N}{N}$, a convergence rate like $\frac{(\ln N)^{2}}{N}$. This means, that in contrast to Section 2 , where the convergence was essentially proportional to $N^{-\frac{1}{2}}$, a much better rate, essentially $N^{-1}$ is achieved.

\section{Numerical Results}

In this section we compute the entropy functional $-\int f(v) \ln f(v) d v$ in 3 different ways. The first one is the coarse graining method described in Section 2.

The second one is the Hermite expansion method from Section 3. In contrast to Section 3 we expand $f$ here in the following series of Hermite functions

$$
f(v)=M_{u, T}(v) \sum_{k=0}^{\infty} \frac{1}{k !} \int f\left(v^{\prime}\right) \tilde{H}_{k}\left(\frac{v^{\prime}-u}{\sqrt{T}}\right) d v^{\prime} \cdot \tilde{H}_{k}\left(\frac{v-u}{\sqrt{T}}\right)
$$

where

$$
M_{u, T}(v):=\frac{1}{(2 \pi T)^{\frac{1}{2}}} \exp \left(-\frac{(v-u)^{2}}{2 T}\right)
$$


with

$$
u=\int v f(v) d v, \quad T=\int(v-u)^{2} f(v) d v .
$$

and $\tilde{H}_{k}$ are the Hermite polynomials defined at the beginning of Section 3. The integration formula reads then for the entropy $\phi(x)=-x \ln x, x>0$

$$
\int \phi(f(v)) d v \sim \sum_{i \in I_{h}^{R}} h \phi\left(\sum_{k=0}^{K} \tilde{a}_{k}\left(\omega_{N}\right) M_{u, T}\left(\xi_{i}\right) \frac{1}{k !} \tilde{H}_{k}\left(\frac{\xi_{i}-u}{\sqrt{T}}\right)\right)
$$

with

$$
\begin{gathered}
\tilde{a}_{k}\left(\omega_{N}\right)=\frac{1}{N} \sum_{j=1}^{N} \tilde{H}_{k}\left(\frac{v_{j}-u}{\sqrt{T}}\right), \\
u=\frac{1}{N} \sum_{j=1}^{N} v_{j}, T=\frac{1}{N} \sum_{j=1}^{N}\left(v_{j}-u\right)^{2} .
\end{gathered}
$$

The third method is derived by the linearization of the entropy functional, taking only deviations from a Maxwellian up to second order into consideration:

$$
-\int f(v) \ln f(v) d v \sim \frac{1}{2} \ln (2 \pi T)+\frac{1}{2}-\frac{1}{2} \int f^{2}(v) M_{u, T}^{-1}(v) d v+\frac{1}{2} .
$$

$T$ and $u$ are computed as above. Hence only the computation of $\int f^{2}(v) M_{u, T}^{-1}(v) d v$ is left. We substitute the above expansion for $f$ and get as in Proposition 3.2 the formula

$$
\int f^{2}(v) M_{u, T}^{-1}(v) d v \sim \sum_{k=0}^{K} \tilde{a}_{k}^{2}\left(\omega_{N}\right) \frac{1}{k !} .
$$

To obtain the f-distributed pointsets $\left\{\left(v_{1}, \ldots, v_{N}\right)\right\}_{N \in \mathbb{N}}$ in $\mathbb{R}$ we transform the points $v_{1}=\frac{1}{2 N}, v_{2}=\frac{3}{2 N}, \ldots, v_{N}=\frac{2 N-1}{2 N}, N \in \mathbb{N}$ to f-distributed ones. The latter are uniformly distributed on $[0,1]$ and we have only to calculate the inverse of the distribution function associated with the density $f$ to get the desired pointsets. We remark that - in contrast to the higher dimensional case - there is no difficulty in computing this transformation here. The number of points is taken between 5 and 200. The number of particles used in numerical calculations for problems in kinetic theory is actually - due to the limitations in computing time and the complexity of the problems - very low, i.e. between 20 and 100 per cell in space.

In Figure 1 and 2 we show the results for the Maxwellian distribution

$$
f(v)=M_{\bar{u}, \bar{T}}(v)=\frac{1}{(2 \pi \bar{T})^{\frac{1}{2}}} \exp \left(-\frac{(v-\bar{u})^{2}}{2 \bar{T}}\right)
$$

for $\bar{T}=1$ and $\bar{u}=0$.

Figure 1 shows the entropy multiplied by -1 . It is computed by the Hermite expansion method in the 2 ways explained above. Expansions with terms up to order 6 respectively 8 are used.

In Figure 2 the computation is done by the coarse graining method with several different numbers of cells. 

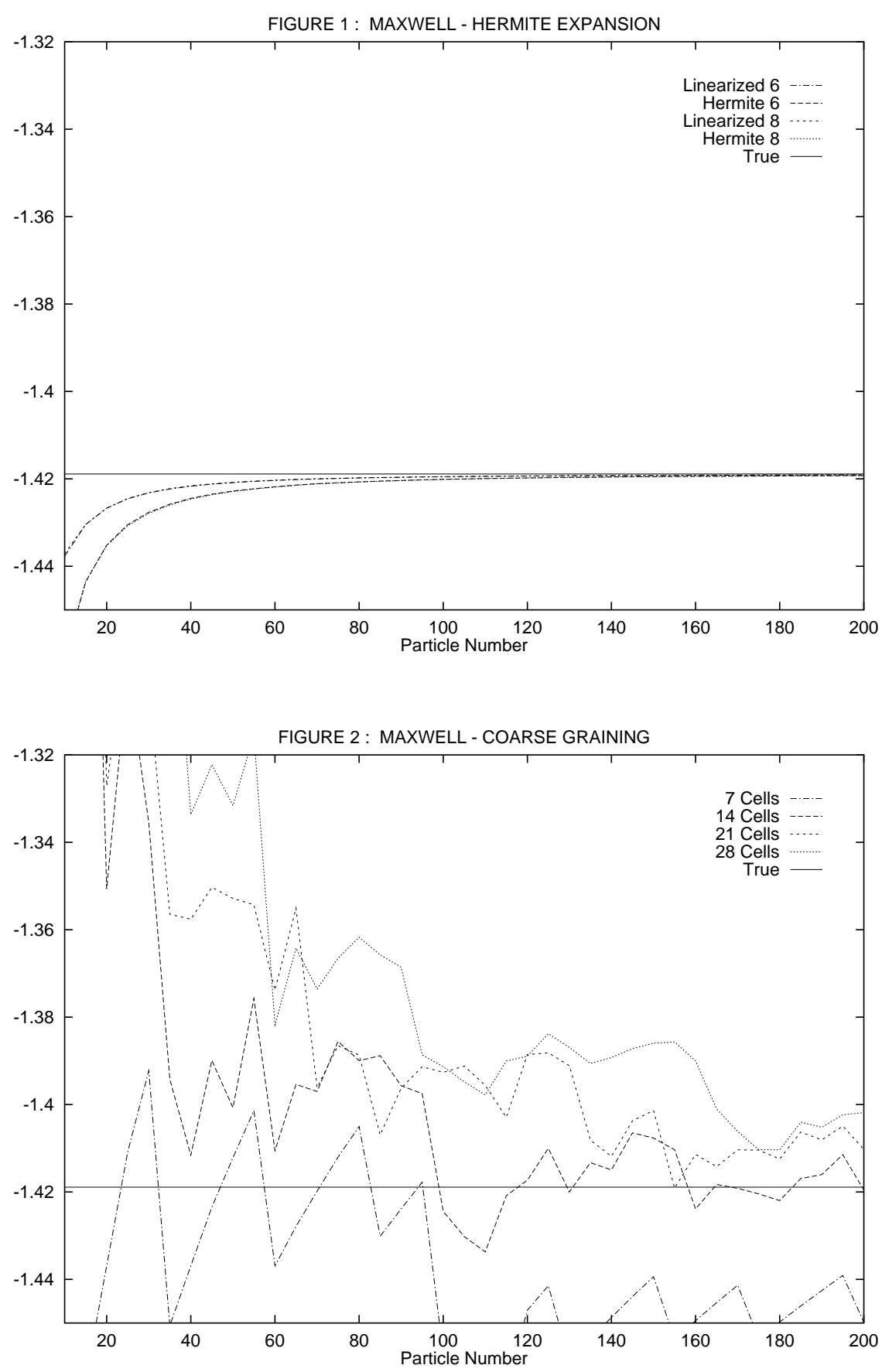

In Figure 3 and 4 the same is shown for the Navier Stokes distribution function

$$
f(v):=F_{\bar{\rho}, \bar{u}, \bar{T}}^{N S, \epsilon}(v):=\bar{\rho} M_{\bar{u}, \bar{T}}(v)\left(1-\epsilon \frac{v-\bar{u}}{\sqrt{\bar{T}}}\left(\frac{(v-\bar{u})^{2}}{\bar{T}}-3\right)\right)
$$

$\forall v$ such that $F_{\bar{\rho}, \bar{u}, \bar{T}}^{N S, \epsilon}(v) \geq 0$ and $f(v):=0$ otherwise. $\bar{\rho}, \bar{u}, \bar{T}$ are chosen in such a way that 


$$
\int\left(\begin{array}{c}
1 \\
v \\
v^{2}
\end{array}\right) F_{\bar{\rho}, \bar{u}, \bar{T}}^{N S, \epsilon)}(v)=\left(\begin{array}{l}
1 \\
0 \\
1
\end{array}\right) .
$$

$\epsilon$ is set equal to 0.1 .
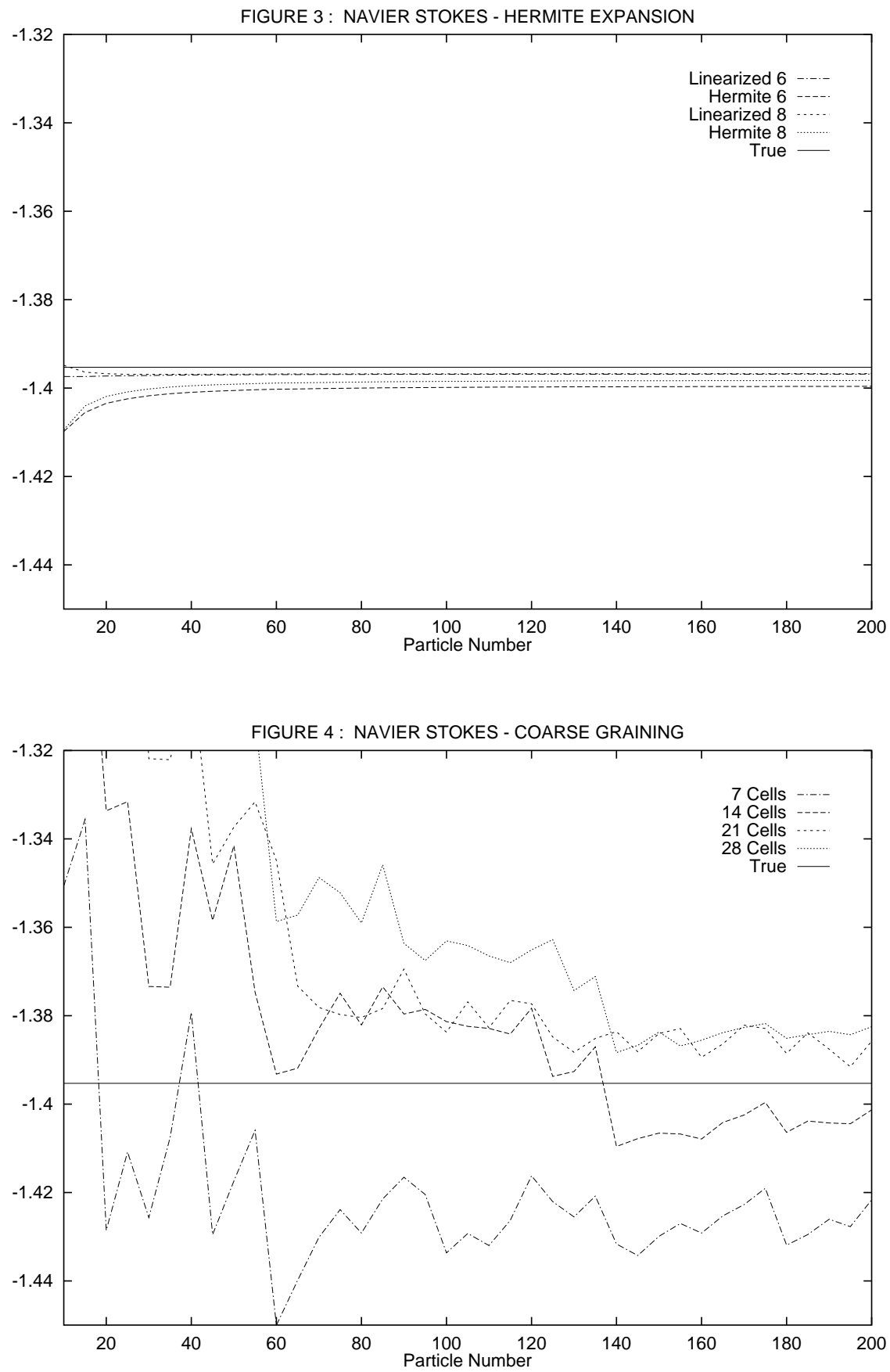
In Figure 5 and 6 we consider a Mott Smith distribution

$$
f(v):=F_{\bar{u}, \bar{T}}^{M S}(v):=\frac{1}{2} \frac{1}{(2 \pi \bar{T})^{\frac{1}{2}}}\left(\exp \left(-\frac{(v-\bar{u})^{2}}{2 \bar{T}}\right)+\exp \left(-\frac{(v+\bar{u})^{2}}{2 \bar{T}}\right)\right)
$$

$\bar{u}$ is chosen equal to 0.8 and $\bar{T}$ in such a way that $\int v^{2} F_{\bar{u}, \bar{T}}^{M S}(v) d v=1$.
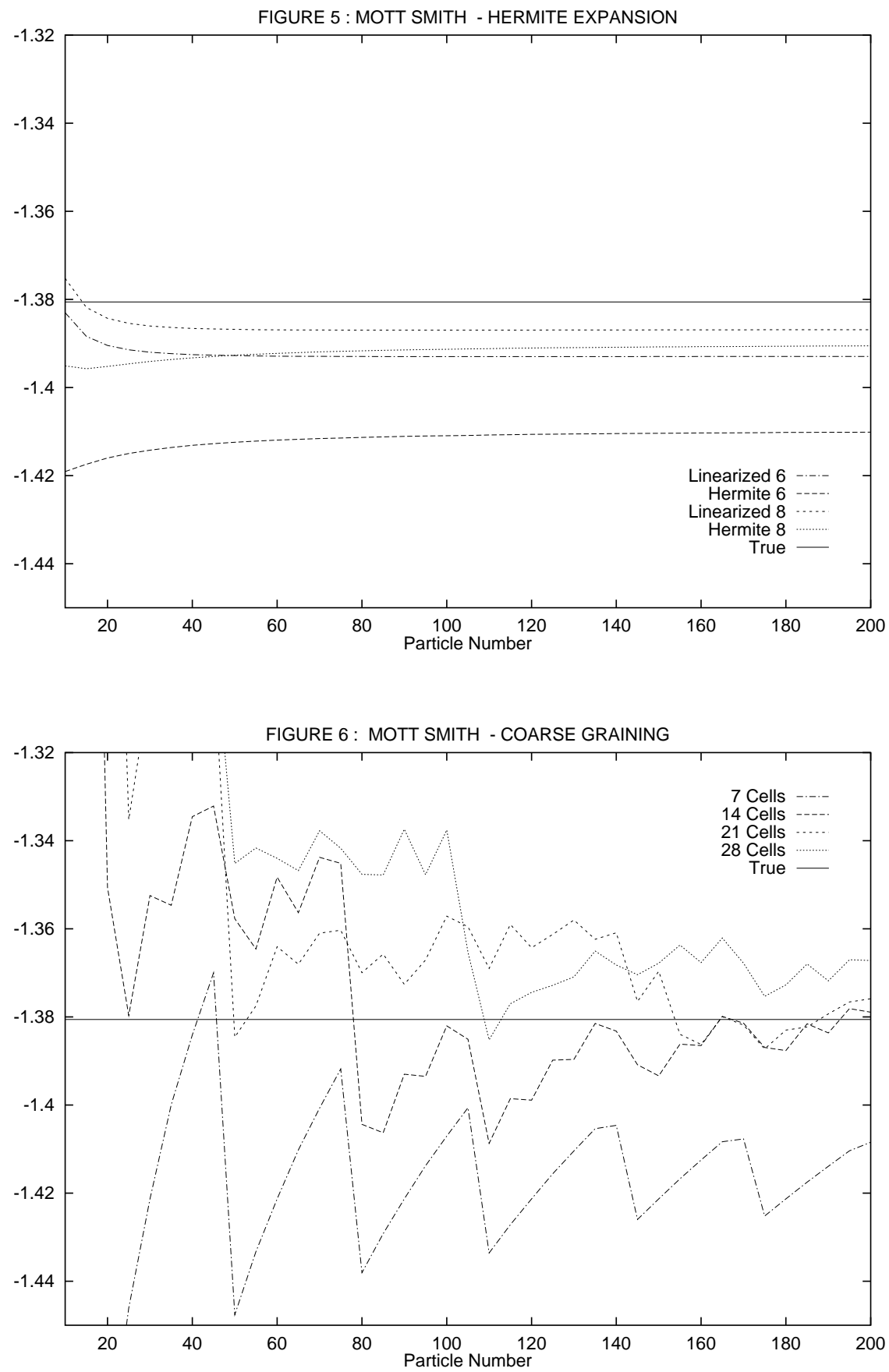
We remark that in contrast to the first two examples the results here are not as good. For larger $\bar{u}$ they are becoming even worse. The above function is not well enough approximated by a finite sum of Hermite polynomials for $\bar{u}$ large.

As remarked in Cercignani [3], the Grad approach of Hermite expanding the distribution function gives inadequate results in strongly nonequilibrium cases, e.g., in shock wave computations. This fits to the above observation for the Mott Smith distribution function, describing the velocity distribution in shock regions.

In general, for fluid dynamic applications, distribution functions describing the behaviour in the bulk of a fluid will fit into the above scheme. In shock and boundary regions however it will certainly not be completely adequate. In thoses cases another class of approximating functions should be chosen. For example in shock regions one may think of approximating the distribution function by linear combinations of Maxwellians, see e.g. Mott Smith [7]. Another obvious disadvantage is the requirement of continuity of the distribution function, which is in general not true for example at a flat boundary. To avoid it classes of piecewise continuous functions might be appropriate, see Cercignani $[3]$.

We note that for all types of density functions considered up to now

$$
\int f(v)\left(\begin{array}{c}
1 \\
v \\
v^{2}
\end{array}\right) d v=\left(\begin{array}{l}
1 \\
0 \\
1
\end{array}\right)
$$

This is also true for the last two figures.

Figures 7 and 8 show the time evolution of the entropy multiplied by -1 for a fixed number of particles $N=70$. The density function is the one dimensional analogue of the BKW solution of the Boltzmann equation with Maxwellian molecules

$$
f(t, v):=\frac{1}{(2 \pi K \bar{T})^{\frac{1}{2}}} \exp \left(-\frac{v^{2}}{2 K \bar{T}}\right) \frac{1}{2}\left(\frac{3 K-1}{K}+\frac{1-K}{K^{2}} \frac{v^{2}}{\bar{T}}\right)
$$

with $K(t)=1-e^{-t}, t \geq \ln \frac{3}{2}, \bar{T}=1$. Again the different methods of computation as before are used. 

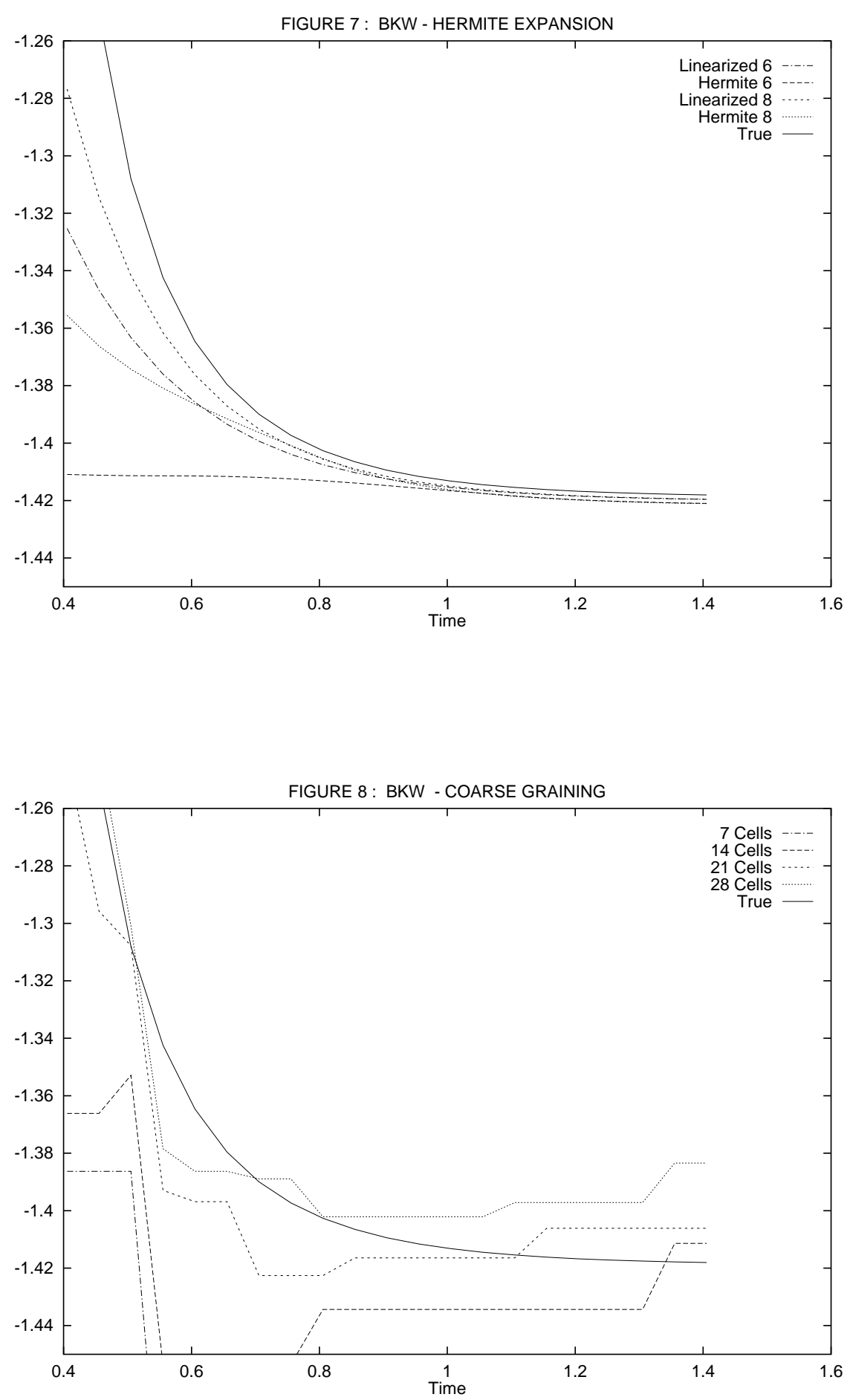

One can see that for situations far away from equilibrium at the beginning of the evolution the true entropy is again not well enough approximated by the Hermite expansion. 
Acknowledgements I am grateful to Prof. H. Neunzert for suggesting this work.

\section{References}

[1] H. Babovsky, R. Illner, A convergence proof for Nanbu's simulation method for the full Boltzmann equation, SIAM J. of Num. Anal. 26, 1, 45, (1989)

[2] G.A. Bird, Molecular gas dynamics, Clarendon Press, Oxford (1976)

[3] C. Cercignani , The Boltzmann Equation and its Applications, Springer (1988)

[4] H. Grad, On the Kinetic Theory of Rarefied Gases, CPAM 2, p.331 (1949)

[5] R. Illner, H. Neunzert, On Simulation Methods for the Boltzmann Equation, TTSP $16(2,3)$, p.141 (1987)

[6] L. Kuipers, H. Niederreiter, Uniform Distribution of Sequences, Wiley, New York (1974)

[7] H.M. Mott Smith, The solutions of the Boltzmann equation for a shock wave, Phys. Rev., 82, 885 (1951)

[8] H. Neunzert, J. Struckmeier, Boltzmann simulation by particle methods, Acta Numerica, 1995 (1994)

[9] H. Neunzert, J. Wick, Die Darstellung von Funktionen mehrerer Variabler durch Punktmengen, Berichte der KFA Jülich 996

[10] J. Struckmeier, F.J. Pfreundt, On the efficiency of simulation methods for the Boltzmann equation on parallel computers, Parallel Computing 19, 103, (1993)

[11] H. Weyl, Über die Gleichverteilung von Zahlen mod 1, Math. Ann.77 (1916) 